\title{
The reliability of perinatal and neonatal mortality rates: differential under-reporting in linked professional registers vs. Dutch civil registers
}

\author{
Sabine Anthony ${ }^{a}$, Karin M. van der Pal-de Bruinn ${ }^{a}$, Wilco C. Graafmans ${ }^{b}$, Caroline A. Dorrepaal ${ }^{a}$, Marion Borkent-Polet ${ }^{c}$, \\ Oscar J. S. van Hemel ${ }^{d}$, Frans H. M. Jansen ${ }^{\mathrm{e}}$ and A. Lya den Ouden ${ }^{\mathrm{a}}$ \\ ${ }^{a}$ Department of Reproduction and Perinatology, TNO Prevention and Health, Leiden, ${ }^{b}$ Faculty of Medicine, Department of Public Health, \\ Erasmus University, Rotterdam, 'Department of Obstetrics, University Medical Center, Utrecht, ${ }^{d}$ Department of Obstetrics and Gynaecology, \\ Reinier de Graaf Teaching Hospital, Delft, 'Juliana Children's Hospital, The Hague, The Netherlands
}

Correspondence:

Sabine Anthony, TNO

Prevention and Health,

Department of Reproduction and Perinatology, PO Box 2215, 2301 CE Leiden, The Netherlands.

E-mail: s.anthony@pg.tno.nl

\section{Summary}

Official Dutch perinatal mortality rates are based on birth and death certificates. These civil registration data are not detailed enough for international comparisons or extensive epidemiological research. In this study, we linked and extrapolated three national, incomplete, professional registers from midwives, obstetricians and paediatricians, containing detailed perinatal information. This linkage and extrapolation resulted in one detailed professional database which is representative of all Dutch births and from which gestational age-specific perinatal mortality rates could be calculated. The reliability of these calculated mortality rates was established by comparing them with the rates derived from the national civil registers. The professional database reported more perinatal deaths and fewer late neonatal deaths than the civil registers. The underreporting in the civil registers amounted to 1.2 fewer perinatal deaths per 1000 births and was most apparent in immature newborns. We concluded that under-reporting of perinatal and neonatal deaths depends on the data source used. Mortality rates for the purpose of national and international comparison should, therefore, be defined with caution. This study also demonstrated that combining different incomplete professional registers can result in a more reliable database containing detailed perinatal information. Such databases can be used as the basis for extensive perinatal epidemiological research.

\section{Introduction}

Perinatal and neonatal mortality rates have been widely used as indicators of the quality of obstetric and neonatal care. They are also used to compare quality of care and health status in different countries. The mortality rates used in these international comparisons, however, often lack comparability. ${ }^{1-5}$ Different definitions are used to determine stillbirths and neonatal deaths, the statutory lower limit for the registration of perinatal deaths varies between countries and there is a large diversity in sources of mortality information. Moreover, information is often lacking as to the completeness and validity of these sources. ${ }^{6}$

In developed countries the rate of perinatal and neonatal deaths is also determined by factors other than quality of care. The proportion of low or very-low-birthweight and of preterm infants, the frequency of congenital malformations (taking into account the frequency of antenatal screening and induced abortions) and the distribution of socioeconomic conditions are all factors that influence the frequency of perinatal mortality. ${ }^{1,-10}$ Therefore, to ensure a fair comparison of national and international perinatal mortality rates, detailed information about such risk factors should be available. Comparisons of mortality rates can then be stratified or standardised for such risk factors, for example by using birthweight or gestational age-specific mortality rates. The Dutch national perinatal statistics derived from the civil registers do not contain such 
detailed information on risk factors for perinatal mortality.

It is mandatory for Dutch citizens to report all births to the local authorities. The notification of deaths is directly related to the permission needed for the obligatory burial or cremation. For each death physicians have to complete a death certificate that is sent to the local authorities. For stillbirths $<24$ weeks of gestation burial or cremation is not obligatory and, therefore, no death certificates are completed. The civil birth and death registers of the local authorities together with the death certificates of the physicians form the basis for the annual vital statistics reported by Statistics Netherlands (CBS). Statistics Netherlands reports on all births and deaths of stillborns of more than 24 weeks of gestation and all liveborns. These reported statistics are the primary source for the Dutch perinatal and neonatal mortality rates. These national birth and death statistics, however, do not contain detailed perinatal information on, for example, risk factors for mortality. Hence, it is impossible to calculate birthweight-standardised mortality rates or perinatal mortality rates according to the World Health Organisation (WHO) definitions using the data from the civil registers. ${ }^{11}$ The WHO definitions use either birthweight or gestational age to define the lower limit for registration of all births. The civil data managed by Statistics Netherlands contain gestational age only for stillbirths and early neonatal deaths and birthweight only for early neonatal deaths. For late neonatal deaths and newborns who stay alive, used in the denominators of these rates, this information is not available. The usefulness of the data derived from the civil registers for perinatal epidemiological research is, therefore, limited. These data must be supplemented by alternative sources of perinatal information.

In The Netherlands, independent midwives, obstetricians and paediatricians register the care they provide in three separate professional databases. Not all professional care providers participate in the registers yet and, therefore, the databases are not complete. These databases contain more detailed information than the civil registers of Statistics Netherlands and can, therefore, constitute the basis for more extensive perinatal epidemiological research.

In the present study, we developed a method for linking and extrapolating these databases in order to create one perinatal database representative of all Dutch births and containing detailed information about pregnancy, birth, puerperium and the newborns. To determine the reliability, we compared the mortality rates calculated using this linked professional database with the mortality rates derived from the civil registers of Statistics Netherlands. Reasons for observed discrepancies and implications for future research using these databases are discussed.

\section{Data and method}

\section{Data}

We used the 1995 data from three Dutch professional registers: the National Perinatal Database for primary care by independent midwives (Landelijke Verloskunde Registratie $1^{\mathrm{e}}$ lijn, LVR-1), the National Perinatal Database for secondary care by obstetricians (Landelijke Verloskunde Registratie $2^{\mathrm{e}}$ lijn, LVR-2) and the National Neonatology Database of paediatricians (Landelijke Neonatologie Registratie, LNR). The database for primary obstetric care by general practitioners (GPs) is still being developed and could not yet be used.

Midwives, obstetricians and paediatricians record information about the perinatal period and information on newborns admitted to a paediatric ward. The National Perinatal Databases (LVR-1 and LVR-2) contain anonymous records of all pregnancies with a gestational age of at least 16 weeks. The National Neonatology Database (LNR) contains anonymous records on all admissions of newborns to paediatric neonatal departments within the first 28 days of life and re-admissions for perinatal problems.

\section{Method}

To calculate perinatal mortality (stillbirths and early neonatal deaths within the first week of life) and neonatal mortality (neonatal deaths within the first month of life), all births registered in the LVR-1 and LVR-2 were combined. In the LVR-1 and LVR-2 deaths are registered up to 7 days after birth. The LNR was added to assess early and late neonatal mortality within 1 month after birth for newborns referred to a paediatric department.

The creation of one linked, perinatal database representative of all births in the Netherlands consisted of two different steps: linkage and extrapolation. The first step was to identify identical child records in the different databases to prevent double counts of births and 
deaths. Births and deaths could be recorded more than once because of frequently occurring referral between the different levels of care, mostly from primary to secondary care in case of complications during pregnancy, labour or puerperium. These pregnancies are often registered both in the LVR-1 and LVR-2. If the newborn is referred to the paediatrician after birth, he or she is also registered in the LNR. Newborns can also be registered more than once in the LNR if neonatal transfer to an intensive care unit or re-admission takes place.

In the second step, the linked professional database was extrapolated to correct for the non-participation of several midwifery practices, non-teaching (level I) hospitals and the GPs. Because non-participation only occurred in pregnancies with low risk of mortality, correction is essential to obtain a representative database of all births and deaths in the Netherlands.

\section{Linkage by identification of duplicate records}

The computerised method used to identify duplicate records and link the three databases is described in brief below. First, the LVR-1 and LVR-2 records were linked by matching based on mother's postal code, mother's date of birth, place of birth (at home or by individual hospital code), child's date of birth, sex of the child and birth order for multiple births. To find duplicate records even if one of these variables was missing or discrepant between records, the search for identical child records was repeated several times, allowing one variable to be discrepant or missing at each run, while including an extra check on birthweight and gestational age. While developing the computerised method for identification of duplicate records, extensive manual checks were performed to determine whether the automated runs correctly marked records as identical or non-identical.

After the identification of duplicate records within the LVR-1 and LVR-2, the newborns in the LNR database were matched with their corresponding LVR record using a method similar to the one described above. Before matching the LNR records with the LVR, different records for the same newborn within the LNR were identified and marked.

Once all duplicate records within the LVR-1, LVR-2 and LNR had been marked, the duplicate records were reduced to single records by aggregating them. The resulting linked professional LVR/LNR database, therefore, contained one record for each newborn.

\section{Adjustment for non-participation by extrapolation}

In 1995, 89\% of all midwifery practices participated in the LVR-1. All university (level III, $n=12$ ) and teaching hospitals (level II, $n=22$ ) participated and $84 \%$ of the non-teaching hospitals (level I, $n=69$ ) participated in the LVR-2. To obtain a database representative of the entire population of births in the Netherlands both in number and in risk profile, the database was extrapolated to $100 \%$ participation by applying a weighting factor to the records. The applied weighting depended on the participation rate of the level of care recorded during delivery.

The missing number of births in the care of GPs was determined by subtracting the extrapolated numbers of liveborns in the linked professional database from the number of liveborns reported in the civil registers from Statistics Netherlands. To determine the additional number of stillbirths and the number of neonatal deaths in GP care, death rates for births under midwifery care were used. Births assisted by GPs are similar to those assisted by midwives as all are lowrisk births.

Neonatal deaths are expected to be under-reported in the LVR-1 and LVR-2 databases as they may occur after referral of the newborn to a paediatric ward. In $1995,50 \%$ of the general paediatric departments (level I/II) and all Neonatal Intensive Care Units (NICU, level III) participated in the LNR. Neonatal deaths reported in the LNR and not in the LVR were also extrapolated to $100 \%$ participation by weighting the records depending on the level of recorded care.

\section{Comparison of mortality rates}

The calculated perinatal and neonatal mortality rates for 1995 were compared with the rates derived from the civil registers of Statistics Netherlands. ${ }^{12}$ As stillbirths $<24$ weeks of gestation are not reported by Statistics Netherlands and are, therefore, not included in the calculated rates, the same selection was applied when calculating the mortality rates from the professional LVR/LNR database. For livebirths, no lower limit was needed.

\section{Results}

In 1995, the LVR-1 database for independent midwives contained 130570 records and the LVR-2 database for obstetricians contained 100887 records, resulting in a 
Table 1. Distribution of level of care and number of births in the linked professional database, before and after extrapolation for nonparticipation (1995)

\begin{tabular}{|c|c|c|c|}
\hline & \multirow{2}{*}{$\frac{\text { Before extrapolation }}{\text { No. of newborns }}$} & \multirow{2}{*}{$\begin{array}{l}\text { Participation } \\
\text { rate }\end{array}$} & \multirow{2}{*}{$\frac{\text { After extrapolation }}{\text { No. of newborns }}$} \\
\hline & & & \\
\hline \multicolumn{4}{|l|}{ Linked professional LVR/LNR database } \\
\hline Primary care (LVR-1) & 61286 & $89 \%$ & 68861 \\
\hline Secondary care (LVR-2), level I & 16061 & $84 \%$ & 19120 \\
\hline Secondary care (LVR-2), level II/III & 18048 & $100 \%$ & 18048 \\
\hline \multicolumn{4}{|l|}{ Combination of care } \\
\hline LVR-1 + LVR-2 level I, linked & 25898 & $84 \%$ & 30831 \\
\hline LVR-1 + LVR-2 level II/III, linked & 16922 & $100 \%$ & 16922 \\
\hline LVR-2 level I, LVR-1 not linked & 14349 & $84 \%$ & 17082 \\
\hline LVR-2 level II/III, LVR-1 not linked & 8405 & $100 \%$ & 8405 \\
\hline Total (including all stillbirths) & 160969 & & 179269 \\
\hline Total (stillbirths $<24$ weeks of gestation excluded) & 160104 & & 178328 \\
\hline Number of births ascribed to GPs ${ }^{a}$ & & & 13472 \\
\hline $\begin{array}{l}\text { Total LVR/LNR } \\
\text { (stillbirths }<24 \text { weeks of gestation excluded) }\end{array}$ & & & 191800 \\
\hline
\end{tabular}

${ }^{\mathrm{a} C a l c u l a t i o n}=($ number of liveborns registered by Statistics Netherlands - liveborns in extrapolated LVR/LNR) + same percentage of stillbirths as for midwifery care $(0.04 \%)$.

combined total of 231457 records. Linkage of these two databases showed that approximately $32 \%$ of all the newborns were registered in the database of both primary (LVR-1) and secondary (LVR-2) obstetric care providers. After excluding the double records by aggregation and excluding records including no actual births (records with only pregnancy or puerperium information), each record in the linked professional LVR database represented one birth ( $n=$ 160969).

\begin{tabular}{lcc}
\hline & LVR/LNR + GPs & Statistics Netherlands \\
\hline Total births $(n)$ & & \\
Stillbirths $<24$ weeks gestation excluded & 191800 & 191735 \\
Stillbirths $<28$ weeks gestation excluded & 191499 & 191474 \\
Stillbirths $(n)$ & & \\
$\geq 24$ week gestation & 1287 & 1222 \\
$\geq 28$ week gestation & 986 & 961 \\
Live births ( $n$ ) & & \\
Total & 190513 & 190513 \\
Early neonatal deaths (1st week) & 762 & 588 \\
Late neonatal deaths (2nd-4th week) & 84 & 189781 \\
Alive after 4 weeks & 189667 & 6.37 \\
Stillbirths per 1000 births & & 5.02 \\
$\geq 24$ weeks gestation & 6.71 & 3.84 \\
$\geq 28$ weeks gestation & 5.15 & 3.09 \\
Neonatal mortality per 1000 live births & 4.44 & 0.76 \\
Early neonatal mortality & 4.00 & \\
Late neonatal mortality & 0.44 & 9.44 \\
Perinatal mortality per 1000 births & & \\
Stillbirths $<24$ weeks gestation excluded & 10.68 & \\
Stillbirths $<28$ weeks gestation excluded & 9.13 & \\
\hline
\end{tabular}

Table 2. Number of deaths and calculated mortality rates from linked and extrapolated professional LVR/LNR database compared with Statistics Netherlands data (1995) 


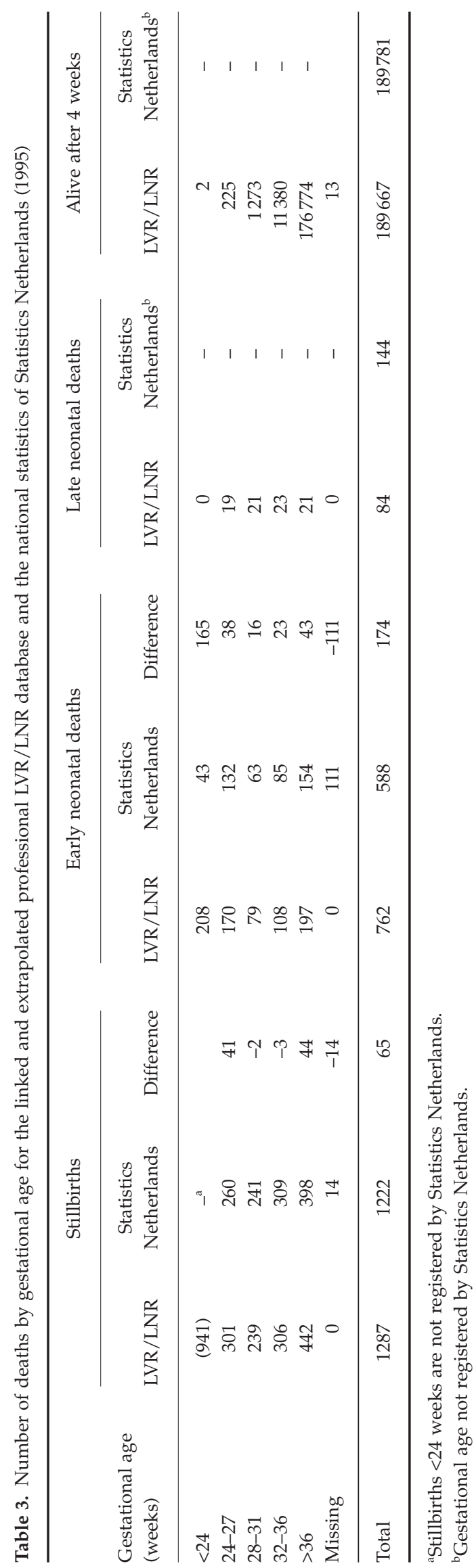

The LNR was matched to the LVR to add the early and late neonatal deaths registered in the LNR but not in the LVR. The LNR of 1995 consisted of 21818 records. Of these records, 20083 are first admissions of newborns to a paediatric department. This number, therefore, corresponds to the number of newborns registered in the LNR of 1995. Of these LNR records, 89\% could be matched to their corresponding LVR record. The main reason for non-linkage of the remaining LNR records was non-participation of a number of obstetrics departments, midwifery practices and GPs.

Linkage of the LNR database to the LVR database added 35 early neonatal deaths and 75 late neonatal deaths registered in the LNR database but not in the LVR database. Apart from six neonatal deaths in a general hospital, all others occurred in one of the NICUs with a complete register.

The number of births by level of care in the linked professional LVR/LNR database before and after extrapolation for non-participating midwifery practices and non-teaching level I hospitals is shown in Table 1. After extrapolation, the linked professional database consisted of 179269 births. For comparison with the civil register statistics, stillbirths $<24$ weeks of gestation were excluded. This resulted in 178328 birth records. The calculated number of births assisted by GPs was 13472 (the number of liveborns reported by Statistics Netherlands minus the number of liveborns in the linked and extrapolated professional database plus the same percentage of stillbirths [0.04\%] as reported for the primary care of midwives).

Table 2 shows the number of stillbirths, early neonatal deaths and late neonatal deaths as well as the perinatal and neonatal mortality rates calculated from both the linked professional database and the civil registers of Statistics Netherlands. The number of stillbirths and early neonatal deaths and, therefore, the calculated mortality rates were higher in the professional LVR/LNR database than in the civil registers, at limits of both 24 and 28 weeks of gestation for stillbirths. For perinatal mortality, the professional database reported 1.2 more deaths per 1000 births than the civil statistics of Statistics Netherlands. The opposite was observed for the late neonatal deaths where the professional LVR/LNR database reported fewer cases than the civil registers (84 vs. 144).

To find a possible explanation for the observed discrepancies between the number of deaths in the professional database and the civil registers, we compared

(C) Blackwell Science Ltd. Paediatric and Perinatal Epidemiology 2001, 15, 306-314 
the gestational age distribution of all the deaths (Table 3). The number of stillbirths in the professional LVR/LNR database was similar to the civil statistics reported by Statistics Netherlands for infants with a gestational age between 28 and 36 weeks. However, the number of reported stillbirths for immature newborns (24-27 weeks of gestation) as well as for fullterm newborns was higher in the professional database. Likewise, a higher number of early neonatal deaths, both registered and after extrapolation, was found in the professional LVR/LNR database. Although the civil registers contain a large number of omissions for the gestational age distribution of early neonatal deaths and are therefore incomplete, this observed difference in early neonatal deaths could be ascribed mainly to the under-registration of immature newborns. There were fewer late neonatal deaths registered in the professional LVR/LNR database than in the civil registers. A distribution by gestational age is not available for these deaths reported by Statistics Netherlands because this information is not registered for these cases. This also applies to the largest category: the newborns who stay alive.

\section{Discussion}

To compare perinatal mortality between countries or between regions, official national birth and death statistics are often used. Birthweight and gestational age distribution, frequency of congenital malformations and related induced abortions, ethnicity and socioeconomic conditions are all factors that influence the perinatal mortality rates of a country or region. In the Dutch civil registration statistics, as in statistics of many other countries, information on these risk factors is either not available at all or not available for all births. In the Netherlands, alternative sources of information have to be used to supplement the national civil registers because these statistics are currently not complete enough to calculate meaningful estimates of, for example, perinatal mortality.

In the Netherlands, detailed perinatal information can be obtained from three separate professional databases in which independent midwives, obstetricians and paediatricians register their care. There are two problems with these databases. First of all, newborns can be registered in more than one database because of referrals between the different care providers. Second, these separate databases do not cover all births in the Netherlands because, as yet, not all providers of obstetric care enter records in the databases. In this study, we developed a method for linking these three professional databases using a linkage key for the detection of duplicate records for the same newborn. In addition, an extrapolation was performed on the linked database for the non-participating care providers. In this extrapolation, the level of the obstetric care provided was taken into account because of the specific under-representation of providers of care for low-risk pregnancies.

Approximately $32 \%$ of the newborns registered in the linked professional database were registered in both primary and secondary care. After linkage and extrapolation for the non-participation of certain midwifery practices and obstetric hospitals, the total number of births in the professional perinatal database was $7 \%$ lower than the total number registered in the civil registers and reported by Statistics Netherlands. As the births assisted by the GPs were not yet registered in the professional database, these $7 \%$ of births were ascribed to the GPs. Estimates of the percentage of births assisted by GPs of $10 \%, 9 \%$ and $7.8 \%$ were reported in 1991, 1993 and 1998..$^{13-15}$ Percentages reported previously reveal a decrease in the number of births assisted by GPs over the years. Our estimate of $7 \%$ confirms this trend. The concurrence of our derived percentage with previously published percentages provides an important check for our linkage and extrapolation methodology.

Two assumptions were made. First, it was assumed that the linkage key used was informative enough to detect most of the duplicate records of newborns. On the other hand, it was also assumed that the key was specific enough to prevent linkage of records belonging to different newborns. Undetected duplicate records could affect the resulting total number of births after extrapolation and thereby the derived percentage of births ascribed to the GPs. Another assumption made in our extrapolation was that non-participating midwives, hospitals and GPs annually assist approximately the same number of births and deaths as participating midwives and hospitals at the same level of obstetric care. For the non-teaching hospitals a check could be performed on the number of births. It was concluded that the distribution of the number of births in the non-participating hospitals was similar to those in the participating nonteaching hospitals. Concerning the deaths, there is no reason to assume that the risk profile of women delivering in participating hospitals or midwifery 
practices is different from the one in non-participating hospitals or practices.

For privacy reasons, the three linked professional databases (LVR-1, LVR-2 and LNR) contain only anonymous records. The development of the linkage procedure was, therefore, complicated and timeconsuming. Algorithms had to be defined to decide when records were similar enough to belong to the same child. The linkage of these databases would be much easier and less time-consuming if every child were to receive a unique identification number at birth. This number could then be used to identify the child on every (computerised) form that is filled out by the different care providers during the perinatal period and also later in life. This would have clear benefits in terms of facilitating epidemiological research. In some of the Nordic countries such identification numbers are available but in most countries linkage of different registers is performed using similar methods as in this study.

To determine the reliability of the linked professional database, the deaths registered in the professional perinatal database were compared with the deaths reported in the civil registers of Statistics Netherlands. More stillbirths and more early neonatal deaths were reported in the professional database. The calculated perinatal mortality rate was 1.2 deaths per 1000 births higher in the linked professional database, indicating an under-registration of $11.7 \%$ in the civil registers.

For both the stillbirths and early neonatal deaths, under-registration was mainly concentrated in the gestational age categories $<28$ weeks. This is close to the lower legal limit for stillbirth registration and at the lower limit of viability. The discrepancies between the linked professional database and the civil registers observed in the other gestational age categories are smaller. Slight differences in the determination and registration of gestational age and time of death between the two data sources can explain part of the differences. Moreover, for the early neonatal deaths, the differences can mostly be explained by missing gestational age data in the civil registers. Gestational age is missing for $20 \%$ of the registered early neonatal deaths in the civil data of Statistics Netherlands. If these deaths are proportionally divided over the defined gestational age categories, the differences between the number of registered deaths disappears for all the gestational age categories except for the lowest gestational age category. The observed over-reporting of full-term stillbirths may, to some extent, be caused by duplicate records which have not been linked. This gestational age category contains the largest number of records, making it more difficult to link all the records correctly, especially as, for the stillbirths, some of the linkage information is often missing.

The discrepancies between the numbers of stillbirths and early neonatal deaths in the lower gestational age groups of the civil registers and the professional database are caused by the confusing rules of birth and death notification around the limit of viability. In the Netherlands, stillbirths with a gestational age $<24$ weeks are not registered and the obligation to register stillbirths with a gestational age of 24-28 weeks is relatively recent (1991). Although birth registration of all liveborns is mandatory, liveborn newborns around the limit of viability that die before birth notification are often not registered in the civil registers. Since 1991 permission of the local authorities is no longer needed for burial or cremation of newborns with a gestational age $<24$ weeks of gestation and, therefore, not all births and deaths of these newborns will be notified. As a result, the vital statistics reported by Statistics Netherlands miss certain deaths as was revealed in this study.

When health care providers want to spare parents the additional burden and costs resulting from obligatory notification, they may be inclined, when gestational age is close to the registration limit, to redefine the gestational age so that registration is no longer obligatory. They may also declare a child to be stillborn under the registration limit instead of liveborn, in case the child was born at the lower limit of viability and died shortly after birth. The reporting of an early neonatal death or stillbirth is, therefore, partly determined by the compliance of the doctors with the legal definitions and partly by the wish of the parents to notify and bury or cremate the child. Thus, registration of a child close to the limit for legal registration depends on emotional, financial, cultural and religious factors. From the literature there is ample evidence that these factors play a major role in the way statutory regulations for the registration of births and deaths are being observed. ${ }^{16,17}$

A trend in under-reporting of perinatal deaths in national statistics, especially of immature newborns, has been reported before. In the Netherlands, two comparisons of local registers with the civil registers of Statistics Netherlands reported an under-registration of perinatal mortality of $14.3 \%$, and at least $8.1 \%$. $^{18,19}$ 
This supports our finding of almost $12 \%$ underreporting. Other countries also report underregistration of perinatal deaths. ${ }^{20-25}$ Scott et al. ${ }^{20}$ described an under-reporting of $10 \%$ of perinatal deaths in Ireland. In Belgium, perinatal deaths were under-reported by $14 \%$ in national statistics. ${ }^{25}$ In the United States, under-reporting of fetal deaths ranging from $7 \%$ to almost $50 \%$ was found, depending on the state registry used..$^{21,22}$ It was shown that the lower the gestational age at death, the smaller the chance of being registered. The Confidential Enquiry into Stillbirths and Deaths in Infancy (CESDI) set up since 1992 in England, Wales and Northern Ireland reports on average $4.6 \%$ fewer deaths than the vital statistics derived from the national birth and death registration system. ${ }^{26}$ The completeness of different kinds of perinatal death registration systems depends on existing incentives, laws and perceptions of viability all resulting in a certain registration practice. ${ }^{27,28}$

Late neonatal deaths (occurring more than 1 week after birth) were under-reported in the linked professional database compared with the civil register statistics. Late neonatal deaths in the professional LVR/LNR database all originate from the neonatal LNR database as the obstetric LVR databases only register care during the first 7 days after birth. In the LNR database, only late neonatal deaths occurring in paediatric departments are registered. Late neonatal deaths occurring at home or in other hospital departments, for example, in the surgical departments, are therefore not registered in the LNR and will not be present in the linked professional LVR/LNR database. This hypothesis was tested and confirmed by studying the underlying cause of the late neonatal deaths in the civil registers. On the basis of the cause of death, the deaths were divided into categories with different probabilities of being registered in the neonatal LNR database. If the death categories with no or little probability of registration in the LNR database were left out of consideration in the civil statistics, the number of late neonatal deaths in the professional database was similar to that in the civil registers.

The comparison made between deaths registered in the linked professional perinatal database and deaths reported in the civil registers revealed differential under-reporting of perinatal and neonatal deaths in the two data sources. The fact that there was an explanation for most of the observed differences confirms that the linkage and extrapolation of the three separate professional perinatal databases resulted in one reli- able database representative of the total number of births in the Netherlands. If its limitations are respected, this linked database can be used for a broad field of perinatal epidemiological research because it contains detailed information for all births about pregnancy, delivery, puerperium and the newborn.

In conclusion, the present study has shown that it is possible to create a representative national perinatal database based on three incomplete professional databases. This perinatal database was derived directly from available data without further data collection. The method of record linkage and the applied extrapolation by level of care to correct for the incompleteness of the database resulting from non-participation, provided a representative perinatal database with more detailed perinatal information than is available at present from the national civil registers. The linked professional perinatal database can provide denominator data for estimates of all kinds of rates for all births. Moreover, it can be used for surveillance, monitoring of trends or detailed national and international comparisons of different obstetric outcomes, taking into account important risk factors. Other countries should also search for and use alternative sources of perinatal information to supplement the existing limited national statistics. Only then will they be able to meet the current increasing requirements for good epidemiological and public health research.

\section{Acknowledgements}

We are indebted to the Royal Dutch Midwifery Organisation (KNOV), the Dutch Association for Obstetrics and Gynaecology (NVOG) and the Paediatric Association of the Netherlands (NVK) and all its members. Without their help and their continuing data collection this work would not have been possible. We thank both PRISMANT Health Care Information and Statistics Netherlands (CBS) who gave us permission to use their perinatal registers.

\section{References}

1 Chalmers I, Macfarlane A. Interpretation of perinatal statistics. In: Topics in Perinatal Medicine. Editor: Wharton B. London: Pitman-Medical, 1980; pp. 1-11.

2 Golding J, Butler NR. Studies of perinatal mortality: contrasts and contradictions. In: Social and Biological Effects on Perinatal Mortality. Vol. 3. Perinatal Analyses. Editor: Golding J. Report on an International Comparative Study 
sponsored by the World Health Organisation, University of Bristol, 1990; pp. 1-19.

3 Gourbin C, Masuy-Stroobant G. Registration of vital data: are live births and stillbirths comparable all over Europe? Bulletin of the World Health Organization 1995; 73:449-460.

4 Keirse MJNC. Perinatal mortality rates do not contain what they purport to contain. Lancet 1984; i:1166-1169.

5 Mugford M. A comparison of reported differences in definitions of vital events and statistics. World Health Statistics Quarterly 1983; 36:201-212.

6 Golding J, Tejeiro A, Rojas Ochoa F. The uses and abuses of national statistics. In: Social and Biological Effects on Perinatal Mortality. Vol. 3. Perinatal Analyses. Editor: Golding J. Report on an International Comparative Study sponsored by the World Health Organisation, University of Bristol, 1990; pp. 355-390.

7 Racin AD, Joyce TJ, Li W, Chiasson MA. Recent declines in New York City infant mortality rates. Pediatrics 1998; 101:682-688.

8 Sachs BP, Fretts RC, Gardner R, Hellerstein S, Wampler NS, Wise $\mathrm{PH}$. The impact of extreme prematurity and congenital anomalies on the interpretation of international comparisons of infant mortality. Obstetrics and Gynaecology 1995; 85:941-946.

9 Wigglesworth JS. Monitoring perinatal mortality. A pathophysiological approach. Lancet 1980; ii:684-686.

10 Lee K, Paneth N, Gartner LM, Pearlman M. The very lowbirth-weight rate: principal predictor of neonatal mortality in industrialized populations. Journal of Pediatrics 1980; 97:759-764.

11 Keirse MJNC. De Nederlandse perinatale sterfte in internationaal perspectief. Nederlands Tijdschrift voor Geneeskunde 1987; 131:905-909.

12 Centraal Bureau voor de Statistiek, Ministerie van Volksgezondheid Welzijn en Sport. Vademecum Gezondheidsstatistiek Nederland 1997. 's-Gravenhage: SDU, 1997.

13 Wiegers TA, van der Zee J, Keirse MJNC. Maternity care in The Netherlands: the changing home birth rate. Birth 1998; 25:190-197.

14 SIG. Verloskunde in Nederland: Grote Lijnen 1989-93. Utrecht: SIG, 1996.

15 Wiegers TA, Hingstman L. Inventarisatie 'Verloskundig actieve huisartsen'. Utrecht: NIVEL, Nederlands Instituut voor Onderzoek van de Gezondheidszorg, 1999.
16 Fenton AC, Field DJ, Mason E, Clarke M. Attitudes to viability of preterm infants and their effect on figures for perinatal mortality. British Medical Journal 1990; 300:434-436.

17 Keirse MJNC. Registratie en betrouwbaarheid van perinatale sterfte in Nederland. Nederlands Tijdschrift voor Geneeskunde 1987; 131:891-895.

18 Doornbos JPR, Nordbeck HJ, Treffers PE. The reliability of perinatal mortality statistics in the Netherlands. American Journal of Obstetrics and Gynecology 1987; 156:1183-1187.

19 de Galan-Roosen AE, Kuijpers JC, Oei YB, van Velzen D, Mackenbach JP. Discrepantie tussen resultaten van registratie van perinatale doodsoorzaken door CBS en door eigen onderzoek in de regio Delft-Westland-Oostland. Nederlands Tijdschrift voor Geneeskunde 1997; 141:237-240.

20 Scott MJ, Ritchie JWK, McClure BG, Reid MM, Halliday HL. Perinatal death recording: time for a change? British Medical Journal 1981; 282:707-710.

21 Harter L, Starzyk P, Frost F. A comparative study of hospital fetal death records and Washington State fetal death certificates. American Journal of Public Health 1986; 76:1333-1334.

22 Goldhaber MK. Fetal death ratios in a prospective study compared to state fetal death certificate reporting. American Journal of Public Health 1989; 79:1268-1270.

23 Hilder L, Alberman E. Monitoring fetal and infant survival using regional birth notification data in north-east London. Journal of Epidemiology and Community Health 1998;52:253-258.

24 Chen L, Sun C, Wu D, Shen M, Lee W. Underregistration of neonatal deaths: an empirical study of the accuracy of infantile vital statistics in Taiwan. Journal of Epidemiology Community Health 1998; 52:289-292.

25 Hertoghe L, de Wals P, Piron M, Bertrand F, Lechat MF. Quality of perinatal death registration. European Journal of Pediatrics 1987; 146:473-476.

26 South East Thames Perinatal Monitoring Unit. Confidential Enquiry into Stillbirths and Deaths in Infancy 1993-95. West Kent Health Authority, South East Thames Perinatal Monitoring Unit, Severoaks, 1997.

27 Sepkowitz S. International rankings of infant mortality and the united states' vital statistics natality data collecting system - failure and success. International Journal of Epidemiology 1995; 24:583-588.

28 Howell EM, Blondel B. International infant mortality rates: bias from reporting differences. American Journal of Public Health 1994; 84:850-852. 